\title{
Gray's Time-Varying Coefficients Model for Posttransplant Survival of Pediatric Liver Transplant Recipients with a Diagnosis of Cancer
}

\author{
Yi Ren, ${ }^{1}$ Chung-Chou H. Chang, ${ }^{1,2,3}$ Gabriel L. Zenarosa, ${ }^{4}$ Heather E. Tomko, ${ }^{5}$ \\ Drew Michael S. Donnell, ${ }^{1}$ Hyung-joo Kang, ${ }^{1,6}$ Mark S. Roberts, ${ }^{2,3,4,5}$ and Cindy L. Bryce ${ }^{2,3,5}$ \\ ${ }^{1}$ Department of Biostatistics, Graduate School of Public Health, University of Pittsburgh, Pittsburgh, PA 15261, USA \\ ${ }^{2}$ Department of Medicine, School of Medicine, University of Pittsburgh, Pittsburgh, PA 15261, USA \\ ${ }^{3}$ Department of Clinical and Translational Science, School of Medicine, University of Pittsburgh, Pittsburgh, PA 15261, USA \\ ${ }^{4}$ Department of Industrial Engineering, Swanson School of Engineering, University of Pittsburgh, Pittsburgh, PA 15261, USA \\ ${ }^{5}$ Department of Health Policy and Management, Graduate School of Public Health, University of Pittsburgh, \\ Pittsburgh, PA 15261, USA \\ ${ }^{6}$ Health Geography Lab/Biostatistics Research Group, Division of Preventive and Behavioral Medicine, \\ University of Massachusetts Medical School, Worcester, MA 01605, USA \\ Correspondence should be addressed to Cindy L. Bryce; bryce99@pitt.edu
}

Received 30 November 2012; Revised 2 March 2013; Accepted 10 April 2013

Academic Editor: Shu-hui Chang

Copyright (C) 2013 Yi Ren et al. This is an open access article distributed under the Creative Commons Attribution License, which permits unrestricted use, distribution, and reproduction in any medium, provided the original work is properly cited.

\begin{abstract}
Transplantation is often the only viable treatment for pediatric patients with end-stage liver disease. Making well-informed decisions on when to proceed with transplantation requires accurate predictors of transplant survival. The standard Cox proportional hazards $(\mathrm{PH})$ model assumes that covariate effects are time-invariant on right-censored failure time; however, this assumption may not always hold. Gray's piecewise constant time-varying coefficients (PC-TVC) model offers greater flexibility to capture the temporal changes of covariate effects without losing the mathematical simplicity of Cox PH model. In the present work, we examined the Cox PH and Gray PC-TVC models on the posttransplant survival analysis of 288 pediatric liver transplant patients diagnosed with cancer. We obtained potential predictors through univariable $(P<0.15)$ and multivariable models with forward selection $(P<0.05)$ for the Cox PH and Gray PC-TVC models, which coincide. While the Cox PH model provided reasonable average results in estimating covariate effects on posttransplant survival, the Gray model using piecewise constant penalized splines showed more details of how those effects change over time.
\end{abstract}

\section{Introduction}

Transplantation is often the only viable treatment for children with end-stage liver disease [1], but the shortage of donor livers means that not every child on the waiting list can receive a transplant. Since 2002, prioritization on the waiting list is determined by the model for end-stage liver disease (MELD)/pediatric end-stage liver disease (PELD) severity score, which allocates organs to the sickest individuals first [2]. However, survival outcomes still vary, suggesting that long-term survival is affected by factors other than illness severity at time of transplant.
For example, posttransplant survival is particularly poor for certain diagnoses such as primary liver malignancies (cancer). Among children transplanted during the MELD/PELD era, disease-specific Kaplan-Meier survival plots indicate that transplant recipients with cancer had significantly lower posttransplant survival rates than those with other diseases (logrank test $P<0.001$ ).

We used this subgroup of transplant recipients to compare two alternative methods for estimating posttransplant survival and its significant covariates. Traditionally, survival models have been developed using Cox proportional Hazards $(\mathrm{PH})$ models [3], but some diseases do not adhere to the 
basic assumption of proportional hazards, implying that the covariate effects are not constant over time. In such cases, an alternative survival model that accounts for varying covariate effects must be used, and we chose Gray's piecewise constant time-varying coefficient (PC-TVC) model [4]. The objective of the paper is to demonstrate that Gray PC-TVC model can provide more flexibility in capturing the temporal dynamics of covariate effects during posttransplant period.

\section{Methods}

2.1. OPTN Data. The organ procurement and transplantation network (OPTN) maintains national-level data on all transplant candidates. We obtained a standard transplant analysis and research (STAR) file and restricted the file to 76,233 adult and pediatric liver transplant candidates listed since the MELD/PELD scoring system was first implemented $(02 / 27 / 2002$ through $06 / 25 / 2010)$. We then removed adults age of 18 years or older $(n=70,506)$. Of the remaining candidates, we excluded 2,252 patients who never received a transplant, who received a multiorgan transplant, or whose transplantation date occurred before listing, leaving a pediatric cohort of 3,471 liver transplant recipients for the posttransplant patient survival analysis. We then selected 288 $(8.3 \%)$ pediatric recipients from the cohort with a diagnosis of cancer at time of transplant as the final cohort.

2.2. Covariates. The following 26 variables are included in our study: recipient age, gender, blood type, AfricanAmerican ethnicity, or other; donor age, gender, blood type, race/ethnicity, donor type (cadaveric, living); recipientdonor blood type compatibility, transplant year, procurement distance, "exceptional" transplant case (indicating medical concerns that are not fully reflected in the candidate's MELD/PELD score), waiting time, laboratory values (albumin, bilirubin, INR, creatinine) at time of transplant, positive cytomegalovirus (CMV) test, transplant center location (based on 11 geographic regions defined by UNOS), allocation type, presence of ascites, split liver; presence of portal vein thrombosis, on ventilator at time of transplant, and previous abdominal surgery.

Among 288 children, one recipient had missing values in recipient age, donor age, donor gender, donor type, transplant year, and ventilator use; 18 recipients did not have serum creatinine values $(6.25 \%)$. Since there is no strong clinical reason to believe that these missing values are related to survival or to other covariates, we treated the missing type as missing completely at random (MCAR) and used completecase analysis in our original paper. We later performed a sensitivity analysis, treating missing type as missing at random (MAR) and rerunning the multivariable Gray's models based on multiple imputed data (5 imputations were used).

Other potential covariates were excluded for myriad reasons, including substantial proportion of missing values (cold ischemia time, growth failure), collinearity (use of life support at time of transplant), and lack of variation within the cancer subgroup (encephalopathy, spontaneous bacterial peritonitis, portal hypertensive bleeding).
2.3. Models. To assess the covariate effects on posttransplant patient survival for the cancer cohort, we used two models in our analysis: Cox PH model and Gray PC-TVC model. Cox $\mathrm{PH}$ model provides the estimated average effects while Gray PC-TVC model provides the estimated temporal effects for the covariates of interest. Detailed specifications of these two models are described below.

2.3.1. Cox Proportional Hazards Model. First, we used the Cox PH model, a semiparametric model commonly used in survival analysis. By assuming that the effect of a covariate is multiplicative with respect to the hazard rate and is constant over time, the model is of the form

$$
h(t \mid \mathbf{X})=h_{0}(t) \exp \left(\boldsymbol{\beta}^{\prime} \mathbf{X}\right)
$$

where $h_{0}(t)$ is an unspecified baseline hazard function at time $t, \mathbf{X}$ is a vector of covariates, and $\boldsymbol{\beta}$ is the same dimensional vector of unknown covariate coefficients.

The coefficients are estimated by maximizing the log partial likelihood function

$$
\begin{array}{r}
\ell(\boldsymbol{\beta}) \\
=\sum_{i=1}^{n} \log \left(\prod_{j \in D_{j}} \exp \left(\boldsymbol{\beta}^{\prime} \mathbf{X}_{j}\right)\right. \\
\times\left[\prod _ { r = 1 } ^ { d _ { i } } \left\{\sum_{j \in R_{i}} \exp \left(\boldsymbol{\beta}^{\prime} \mathbf{X}_{j}\right)\right.\right. \\
\left.\left.\left.\quad-\frac{r-1}{d_{j}} \sum_{j \in D_{i}} \exp \left(\boldsymbol{\beta}^{\prime} \mathbf{X}_{j}\right)\right\}\right]^{-1}\right),
\end{array}
$$

where $i$ denotes $n$ distinct event times; $\mathbf{X}_{i}$ is the covariate vector of the individual who experienced the event at $t_{i} ; R_{i}$ is the risk set at time $t_{i} ; D_{i}$ is the event set at time $t_{i}$; and $d_{i}$ is the number of events occurred at time $t_{i}$. Here we used the Efron method [5] to adjudicate tied failure times. Note that the model implies the property of proportional hazards, which needs to be tested.

\subsubsection{Gray Piecewise Constant Time-Varying Coefficients} Model. Gray PC-TVC model is an extension of the Cox PH model. By using a penalized smoothing spline function, Gray PC-TVC model can be used to examine the proportional hazards assumption and to estimate time-varying covariate effects for right-censored data. The model specifies the hazards with the form

$$
h(t \mid \mathbf{X})=h_{0}(t) \exp \left(\boldsymbol{\beta}(t)^{\prime} \mathbf{X}\right),
$$

where $\boldsymbol{\beta}_{j}(t)=\sum_{k} \alpha_{j k} B_{j k}(t)$ is the $j$ th element of $\boldsymbol{\beta}(t)$ and this spline function represents the time-varying coefficient of the $j$ th covariate; $B_{j k}(t), k=1, \ldots, K$ is a set of $B$-spline basis functions; $\alpha_{j k}$ is the corresponding basis coefficient. 
The $B$-spline basis functions are determined by the number of knots and their locations. Knot locations are usually chosen at the times of failure and with roughly equal amounts of failures in between two knots. Under Gray PC-TVC model, the timevarying coefficients are assumed to be constant in between two knots; that is, $\boldsymbol{\beta}_{j}(t)$ is constant for $t \in\left[\tau_{k}, \tau_{k+1}\right)$ where $\tau_{k}$ is the $k$ th knot, $\tau_{1}=0$, and $\tau_{K+1}=T$ represents the maximum observed time of failure. The right-continuous step functions of time with jumps may occur at any internal knots [6].

To estimate the unknown parameters, a penalty function is added to the log partial likelihood function to prevent overfitting of the data. As for cubic splines, the penalty function has the form

$$
\frac{1}{2} \lambda_{j} \int\left[\boldsymbol{\beta}_{j}^{\prime \prime}(s)\right]^{2} d s
$$

where $\lambda_{j}$ is the smoothing parameter indicating the smoothness and $\boldsymbol{\beta}_{j}^{\prime \prime}(s)$ is the second derivative of $\boldsymbol{\beta}_{j}(s)$. The penalty function helps to control the smoothness of the fitted curve through $\lambda_{j}$. When $\lambda_{j}$ reduces to zero, there is no penalty applied. The larger the $\lambda_{j}$, the smoother the curve. The smoothing parameters are usually solved by specifying degrees of freedom. Cubic spline functions tend to be unstable in the right tail of distribution when right censoring yields sparse failure times [4, 7]. In addition to cubic splines, quadratic splines and piecewise constant spline functions can also be applied. The piecewise constant function has the penalty with the form

$$
\frac{1}{2} \lambda_{j} \sum_{k=2}^{K+1}\left(\alpha_{j k}-\alpha_{j, k-1}\right)^{2}
$$

The basis parameters $\alpha$ are estimated by maximizing the penalized log partial likelihood function

$$
\ell_{p}(\alpha)=\ell(\alpha)-\frac{1}{2} \lambda_{j} \sum_{k=2}^{K+1}\left(\alpha_{j k}-\alpha_{j, k-1}\right)^{2},
$$

where $\ell(\alpha)$ is the standard log partial likelihood of Cox model. The penalty function shrinks the size of the jumps at each internal knot in the step functions.

There are two hypotheses of interest: the hypothesis that the $j$ th covariate has no overall effect $\left(H_{0}: \alpha_{j k}=0\right.$ for all knots $k$ ) and the hypothesis that the $j$ th covariate satisfies the condition of proportional hazards $\left(H_{0}: \alpha_{j k}=\alpha_{j 1}\right.$ where $\alpha_{j 1} B_{j 1}$ is a linear term in the $j$ th covariate coefficient).

There are several conventional methods to check the proportional hazards assumption. For instance, we can create time-covariate interactions and include them in the model with other covariates. Alternatively, we can use graphic methods such as checking the Schoenfeld residual plot. Gray PCTVC model offers a method of checking the PH assumption by testing whether all piecewise constant coefficients are the same throughout the follow-up time period. It is worth noting that the order and knots of penalized spline functions can be changed based on the characteristics of the data to suit different conditions. After variable selection, a mixed effect analysis can be accomplished by specifying time-independent variables and time-varying variables. The advantage of Gray PC-TVC model is its flexibility on estimating covariate effects, because it can directly capture the temporal changes of covariates when the assumption of proportional hazards is not satisfied.

2.4. Statistical Analysis. The outcome is posttransplant survival, measured from time of transplantation to death. Recipients who were retransplanted, truncated due to administrative censoring, or lost to followup were subject to right censoring in the analysis.

The selection of explanatory variables in predicting posttransplant survival consists of two steps, univariable selection and multivariable selection. In the univariable selection, each potential covariate specified in the list above was individually fitted using Gray PC-TVC model with 4 degrees of freedom. The number of degrees of freedom used was suggested by Gray [4]. Dummy variables were created at each level of the categorical variables (recipient blood type, donor race/ethnicity and blood type, allocation type, and transplant center location) except for the reference category. Variables with significance at the level of 0.15 were then fitted into the multivariable Gray PC-TVC model to obtain final set of predictors using the forward selection with entry $P$ value less than or equal to 0.05 . We used the same final set of covariates to refit the data using Cox $\mathrm{PH}$ model. All data management and data analyses were implemented in SAS version 9.2 (SAS Institute, Cary, NC, USA) and R version 2.10.0. The Gray PC-TVC models were fit using package coxspline (http://cran.r-project.org/) in R.

\section{Results}

The descriptive statistics for the covariates considered in the univariable models are presented in Table 1 . These statistics are shown for all transplant recipients $(n=288)$ and are also broken down by patients who were alive $(n=237)$ and those who died $(n=51)$ during the posttransplant follow-up period. Median follow-up time of all recipients was 612.5 days (1.68 years).

In the overall sample of 288 recipients, there were 11 recipients with blood type $\mathrm{AB}$, only one of whom died. The number of days of posttransplant survival and the vital status for these 11 recipients are provided in Appendix A. KaplanMeier survival estimates of the posttransplant survival time between those with blood type $\mathrm{AB}$ and those with other blood types (Appendix A) show that recipients with other blood types died faster compared to those with blood type AB. For blood type $\mathrm{AB}$ group, the only jump on the survival function occurred at follow-up day 616 when the recipient died after two subjects were right-censored. The two survival curves do not cross, and the recipients with blood type $A B$ seem to have higher overall survival than that of those with other blood types, but the difference between the two curves is not significant (logrank test $P=0.336$ ).

We also estimated Kaplan-Meier survival curves by donor blood type (Appendix B) and found that survival curves 
TABLE 1: Characteristics of the covariates considered in the univariable models.

\begin{tabular}{|c|c|c|c|}
\hline \multirow{2}{*}{ Characteristics } & \multirow{2}{*}{ All recipients $(N=288)$} & \multicolumn{2}{|c|}{ Patient outcome } \\
\hline & & Alive $(N=237)$ & $\operatorname{Died}(N=51)$ \\
\hline \multicolumn{4}{|l|}{ Recipient characteristics } \\
\hline \multicolumn{4}{|l|}{ Demographics } \\
\hline Age, median, mean \pm SD (years) ${ }^{*}$ & $2.00,4.36 \pm 4.83$ & $2.00,4.12 \pm 4.82$ & $4.00,5.51 \pm 4.78$ \\
\hline \multicolumn{4}{|l|}{ Gender, no. (\%) } \\
\hline Female & $121(42.01)$ & $98(41.35)$ & $23(45.10)$ \\
\hline Male & $167(57.99)$ & $139(58.65)$ & $28(54.90)$ \\
\hline \multicolumn{4}{|l|}{ Race/ethnicity, no. (\%) } \\
\hline Black & $24(8.33)$ & $20(8.44)$ & $4(7.84)$ \\
\hline Nonblack & $264(91.67)$ & $217(91.55)$ & $47(92.16)$ \\
\hline \multicolumn{4}{|l|}{ Medical/clinical covariates } \\
\hline \multicolumn{4}{|l|}{ Blood type, no. (\%) } \\
\hline A & $106(36.81)$ & $91(38.40)$ & $15(29.41)$ \\
\hline $\mathrm{AB}$ & $11(3.82)$ & $10(4.22)$ & $1(1.96)$ \\
\hline $\mathrm{B}$ & $38(13.19)$ & $32(13.50)$ & $6(11.76)$ \\
\hline $\mathrm{O}$ & $133(46.18)$ & $104(43.88)$ & $29(56.86)$ \\
\hline \multicolumn{4}{|l|}{ On ventilator, no. (\%) } \\
\hline Yes & $16(5.56)$ & $10(4.22)$ & $6(11.76)$ \\
\hline No & $271(94.10)$ & $226(95.36)$ & $45(88.24)$ \\
\hline Unknown & $1(0.35)$ & $1(0.42)$ & $0(0.00)$ \\
\hline \multicolumn{4}{|l|}{ Laboratory values, median, mean \pm SD } \\
\hline Albumin $(\mathrm{g} / \mathrm{dL})$ & $3.80,3.66 \pm 0.74$ & $3.80,3.70 \pm 0.71$ & $3.60,3.48 \pm 0.83$ \\
\hline Bilirubin (mg/dL) & $0.50,2.06 \pm 5.91$ & $0.40,1.63 \pm 4.53$ & $0.70,4.11 \pm 9.92$ \\
\hline Serum creatinine $(\mathrm{mg} / \mathrm{dL})^{\dagger}$ & $0.40,0.45 \pm 0.26$ & $0.40,0.42 \pm 0.23$ & $0.55,0.59 \pm 0.37$ \\
\hline INR & $1.10,1.32 \pm 0.87$ & $1.10,1.30 \pm 0.91$ & $1.20,1.39 \pm 0.64$ \\
\hline \multicolumn{4}{|l|}{ Presence of ascites, no. (\%) } \\
\hline Yes & $38(13.19)$ & $27(11.39)$ & $11(21.57)$ \\
\hline No & $166(57.64)$ & $139(58.65)$ & $27(52.94)$ \\
\hline Unknown & $84(29.17)$ & $71(29.96)$ & $13(25.49)$ \\
\hline \multicolumn{4}{|l|}{ Presence of portal vein thrombosis, no. (\%) } \\
\hline Yes & $13(4.51)$ & $11(4.64)$ & $2(3.92)$ \\
\hline No & $265(92.01)$ & $217(91.56)$ & $48(94.12)$ \\
\hline Unknown & $10(3.47)$ & $9(3.80)$ & $1(1.96)$ \\
\hline \multicolumn{4}{|l|}{ Previous abdominal surgery, no. (\%) } \\
\hline Yes & $124(43.06)$ & 99 (41.77) & $25(49.02)$ \\
\hline No & $145(50.35)$ & $123(51.90)$ & $22(43.14)$ \\
\hline Unknown & $19(6.59)$ & $15(6.33)$ & $4(7.84)$ \\
\hline \multicolumn{4}{|l|}{ Positive cytomegalovirus (CMV) test, no. (\%) } \\
\hline Yes & $81(28.13)$ & $60(25.32)$ & $21(41.18)$ \\
\hline No & $207(71.88)$ & $177(74.68)$ & $30(58.82)$ \\
\hline \multicolumn{4}{|l|}{ Other characteristics } \\
\hline \multicolumn{4}{|l|}{ Donor type, no. (\%) } \\
\hline Deceased & $256(88.89)$ & $210(88.61)$ & $46(90.20)$ \\
\hline Living & $31(10.76)$ & $26(10.97)$ & $5(9.80)$ \\
\hline Unknown & $1(0.35)$ & $1(0.42)$ & $0(0.00)$ \\
\hline Donor age, median, mean $\pm \mathrm{SD}$ (years) ${ }^{\ddagger}$ & $14.00,15.35 \pm 14.53$ & $12.00,14.40 \pm 14.07$ & $17.00,19.75 \pm 15.92$ \\
\hline \multicolumn{4}{|l|}{ Donor gender, no. (\%) } \\
\hline Female & $112(38.89)$ & $90(37.97)$ & $22(43.14)$ \\
\hline Male & $175(60.76)$ & $146(61.60)$ & $29(56.86)$ \\
\hline Unknown & $1(0.35)$ & $1(0.42)$ & $0(0.00)$ \\
\hline
\end{tabular}


TABLe 1: Continued.

\begin{tabular}{|c|c|c|c|}
\hline \multirow{2}{*}{ Characteristics } & \multirow{2}{*}{ All recipients $(N=288)$} & \multicolumn{2}{|c|}{ Patient outcome } \\
\hline & & Alive $(N=237)$ & $\operatorname{Died}(N=51)$ \\
\hline \multicolumn{4}{|l|}{ Donor race/ethnicity, no. (\%) } \\
\hline White & $152(52.78)$ & $129(54.43)$ & $23(45.10)$ \\
\hline Black & $51(17.71)$ & $42(17.72)$ & $9(17.65)$ \\
\hline Hispanic & $70(24.31)$ & $56(23.63)$ & $14(27.47)$ \\
\hline Asian & $12(4.17)$ & $7(2.95)$ & $5(9.80)$ \\
\hline Other & $3(1.04)$ & $3(1.27)$ & $0(0.00)$ \\
\hline \multicolumn{4}{|l|}{ Donor blood type, no. (\%) } \\
\hline $\mathrm{A}$ & $85(29.51)$ & $76(32.07)$ & $9(17.65)$ \\
\hline $\mathrm{AB}$ & $2(0.69)$ & $0(0.00)$ & $2(3.92)$ \\
\hline $\mathrm{B}$ & $21(7.29)$ & $18(7.59)$ & $3(5.88)$ \\
\hline $\mathrm{O}$ & $179(62.15)$ & $142(59.92)$ & $37(72.55)$ \\
\hline Unknown & $1(0.35)$ & $1(0.42)$ & \\
\hline \multicolumn{4}{|l|}{ ABO compatible, no. (\%) } \\
\hline Yes & $283(98.26)$ & $233(98.31)$ & $50(98.04)$ \\
\hline No & $4(1.39)$ & $3(1.27)$ & $1(1.96)$ \\
\hline Unknown & $1(0.35)$ & $1(0.42)$ & $0(0.00)$ \\
\hline \multicolumn{4}{|l|}{ Transplantation-related characteristics } \\
\hline \multicolumn{4}{|c|}{ Active exception at time of transplant, no. (\%) } \\
\hline Yes & $128(44.44)$ & $109(45.99)$ & $19(37.25)$ \\
\hline No & $160(55.56)$ & $128(54.01)$ & $32(62.75)$ \\
\hline Unknown & $0(0.00)$ & $0(0.00)$ & $0(0.00)$ \\
\hline \multicolumn{4}{|l|}{ Transplant year, no. (\%) } \\
\hline 2002 & $16(5.56)$ & $10(4.22)$ & $6(11.76)$ \\
\hline 2003 & $18(6.25)$ & $15(6.33)$ & $3(5.88)$ \\
\hline 2004 & $41(14.24)$ & $32(13.50)$ & $9(17.65)$ \\
\hline 2005 & $41(14.24)$ & $32(13.50)$ & $9(17.65)$ \\
\hline 2006 & $35(12.15)$ & $27(11.39)$ & $8(15.69)$ \\
\hline 2007 & $33(11.46)$ & $26(10.97)$ & $7(13.73)$ \\
\hline 2008 & $47(16.32)$ & $40(16.88)$ & $7(13.73)$ \\
\hline 2009 & $32(11.11)$ & $32(13.50)$ & $0(0.00)$ \\
\hline 2010 & $24(8.33)$ & $22(9.28)$ & $2(3.92)$ \\
\hline Unknown & $1(0.35)$ & $1(0.42)$ & $0(0.00)$ \\
\hline \multicolumn{4}{|l|}{ Center location (region), no. (\%) } \\
\hline (1) CT, ME, MA, NH, RI & $12(4.17)$ & $10(4.22)$ & $2(3.92)$ \\
\hline (2) DC, DE, MD, NJ, PA, WV & $49(17.01)$ & $40(16.88)$ & $9(17.65)$ \\
\hline (3) AL, AR, FL, GA, LA, MS, PR & $27(9.38)$ & $22(9.28)$ & $5(9.80)$ \\
\hline (4) OK, TX & $20(6.94)$ & $15(6.33)$ & $5(9.80)$ \\
\hline (5) AZ, CA, NV, NM, UT & $75(26.04)$ & $66(27.85)$ & $9(17.65)$ \\
\hline (6) AK, HI, ID, MT, OR, WA & $8(2.78)$ & $6(2.53)$ & $2(3.92)$ \\
\hline (7) IL, MN, ND, SD, WI & $25(8.68)$ & $18(7.59)$ & $7(13.73)$ \\
\hline (8) CO, IA, KS, MO, NE, WY & $20(6.94)$ & $16(6.75)$ & $4(7.842)$ \\
\hline (9) NY, VT & $14(4.86)$ & $12(5.06)$ & $2(3.92)$ \\
\hline (10) IN, MI, OH & $27(9.38)$ & $23(9.70)$ & $4(7.84)$ \\
\hline (11) KY, NC, SC, TN, VA & $11(3.82)$ & $9(3.80)$ & $2(3.92)$ \\
\hline \multicolumn{4}{|l|}{ Allocation type, no. (\%) } \\
\hline Local & $134(46.53)$ & $106(44.73)$ & $28(54.90)$ \\
\hline Regional & $106(36.81)$ & $87(36.71)$ & $19(37.25)$ \\
\hline Other & $47(16.32)$ & 43 (18.14) & $4(7.84)$ \\
\hline Unknown & $1(0.35)$ & $1(0.42)$ & $0(0.00)$ \\
\hline
\end{tabular}


TABle 1: Continued.

\begin{tabular}{|c|c|c|c|c|}
\hline \multirow{2}{*}{ Characteristics } & & \multirow{2}{*}{ All recipients $(N=288)$} & \multicolumn{2}{|c|}{ Patient outcome } \\
\hline & & & Alive $(N=237)$ & $\operatorname{Died}(N=51)$ \\
\hline \multirow{2}{*}{\multicolumn{2}{|c|}{$\begin{array}{l}\text { Procurement distance, median, mean } \pm \mathrm{SD}(\text { miles })^{\S} \\
\text { Partial or split donor organ, no. }(\%)\end{array}$}} & $156.00,300.36 \pm 411.36$ & $169.00,313.10 \pm 421.48$ & $89.00,241.43 \pm 358.70$ \\
\hline & & & & \\
\hline \multicolumn{2}{|c|}{$\begin{array}{l}\text { Partial or split } \\
\text { Partial or spitt donor organ, no. (\%) }\end{array}$} & $109(37.85)$ & $88(37.13)$ & $21(41.18)$ \\
\hline \multicolumn{2}{|l|}{ Whole } & $178(61.81)$ & $148(62.45)$ & $30(58.82)$ \\
\hline \multicolumn{2}{|l|}{ Unknown } & $1(0.35)$ & $1(0.42)$ & $0(0.00)$ \\
\hline \multicolumn{2}{|c|}{ Waiting time, median, mean \pm SD (days) } & $29.00,45.35 \pm 74.38$ & $28.00,48.00 \pm 80.87$ & $30.00,33.04 \pm 26.42$ \\
\hline \multicolumn{5}{|c|}{$\begin{array}{l}\text { SD: standard deviation. } \\
{ }^{*} \text { The age at time of transplant of one child (alive) was missing. } \\
{ }^{\dagger} \text { Serum creatinine values were missing for } 18 \text { children: } 15 \text { alive and } \\
{ }^{\ddagger} \text { The age of one donor was missing. } \\
{ }^{\$} \text { Procurement distance values were missing for one child (alive). }\end{array}$} \\
\hline \multicolumn{5}{|c|}{$\begin{array}{l}\text { TABLE 2: Estimated log hazard ratios for testing overall covariate effects and the test results of nonproportionality (nonprop) using Cox } \\
\text { proportional hazards (PH) model and Gray piecewise constant time-varying coefficients (PC-TVC) model. }\end{array}$} \\
\hline \multirow{2}{*}{ Covariate } & \multicolumn{2}{|c|}{$\mathrm{Cox} \mathrm{PH}$} & \multicolumn{2}{|c|}{ Gray PC-TVC } \\
\hline & Log hazard ratio (95\% CI) & $P$ value & $P$ value & Nonprop ${ }^{*} P$ value \\
\hline \multicolumn{5}{|l|}{ Donor blood type } \\
\hline $\mathrm{A}$ & $-0.711(-1.484,0.062)$ & 0.071 & 0.163 & 0.498 \\
\hline B & $-0.343(-1.534,0.847)$ & 0.572 & 0.679 & 0.480 \\
\hline $\mathrm{AB}$ & $0.482(-1.208,2.171)$ & 0.576 & 0.001 & 0.001 \\
\hline Serum creatinine $(\mathrm{mg} / \mathrm{dL})$ & $1.635(0.695,2.575)$ & 0.001 & 0.001 & 0.527 \\
\hline On ventilator & $0.828(-0.166,1.821)$ & 0.102 & 0.002 & 0.012 \\
\hline Positive CMV & $0.686(0.096,1.275)$ & 0.023 & 0.017 & 0.163 \\
\hline Female gender & $-0.143(-0.731,0.445)$ & 0.633 & 0.045 & 0.007 \\
\hline
\end{tabular}

${ }^{*}$ Null hypothesis: the proportional hazards $(\mathrm{PH})$ assumption is not violated.

for the four blood types ( $\mathrm{A}, \mathrm{B}, \mathrm{AB}$, and $\mathrm{O}$ ) were not parallel. The Tarone-Ware test indicates a significant difference of posttransplant survival rates among donor blood types (Tarone-Ware Chi-square statistic $=8.0053$ with 3 degrees of freedom, $P=0.046$ ), with donor blood type $\mathrm{O}$ having a lower posttransplant survival rate than other donor blood types.

Similarly, only 16 recipients used ventilator at the time of transplant and 6 (37.5\%) of them died. Given the details in Appendix C, the overall survival for recipients who used ventilator at time of transplant is lower than those who did not (Tarone-Ware test $P=0.001$ ). This indicates that the recipients who used ventilator are transplanted with a worse health condition than those who did not and are unlikely to survive for a long period.

After fitting univariable Gray PC-TVC models, covariates that were statistically significant at the level of 0.15 included recipient characteristics (age, female gender, race (recoded as black versus non-black); laboratory values (albumin, bilirubin, creatinine) at time of transplant; positive CMV; use of a ventilator at time of transplant; presence of ascites at transplant); donor characteristics (age, blood type, race/ethnicity); and recipient-donor blood type compatibility.

Based on these results from the univariable models, significant covariates were then included in the forward selection procedure with entry $P$-value of 0.05 to obtain the final multivariable Gray PC-TVC model. Starting with the most significant, explanatory variables were sequentially added to the model until none of the remaining variables was significant $(P<0.05)$. The final multivariable model included 5 covariates: donor blood type, recipient creatinine at time of transplant, use of a ventilator at time of transplant, positive $\mathrm{CMV}$, and recipient gender. We checked the two-way interactions for the final multivariable models, but none of the interaction terms was significant at level of 0.05 . We then performed a Cox $\mathrm{PH}$ model with these 5 variables.

Table 2 summarizes the estimation and hypothesis testing results for both of these models. Beginning with the Cox $\mathrm{PH}$ model, the table presents the estimated average coefficients (log hazard ratio) with 95\% confidence intervals, as well as $P$-values of testing the significance of the average effect. Based on these results, only creatinine $(P=0.001)$ and positive CMV $(P=0.023)$ had significant average effects on posttransplant survival. Recipients with higher creatinine levels had increased risk of death; likewise, recipients with positive CMV had higher risks of death than those testing negative.

The remainder of the table presents results from the Gray PC-TVC model, with $P$-values indicating (1) the overall effect of the covariate on posttransplant survival and (2) whether the covariate violates the proportional hazards assumption. 
TABLE 3: Estimated time-varying and time-invariant coefficients and $P$ values for testing overall covariate effects and proportional hazards.

\begin{tabular}{|c|c|c|c|c|}
\hline \multirow{2}{*}{ Covariate } & \multicolumn{2}{|c|}{ Log hazard ratio } & \multirow{2}{*}{ Overall $P$ value } & \multirow{2}{*}{ Nonprop $P$ value } \\
\hline & Min & $\operatorname{Max}$ & & \\
\hline \multicolumn{5}{|l|}{ Donor blood type } \\
\hline A & -1.081 & -0.333 & 0.140 & 0.481 \\
\hline B & -0.900 & 0.208 & 0.700 & 0.510 \\
\hline $\mathrm{AB}$ & -2.741 & 5.370 & 0.001 & 0.001 \\
\hline $\begin{array}{l}\text { Serum creatinine } \\
(\mathrm{mg} / \mathrm{dL})\end{array}$ & \multicolumn{2}{|c|}{1.622} & $<0.001$ & \\
\hline On ventilator & -0.844 & 2.364 & 0.002 & 0.011 \\
\hline Positive CMV & \multicolumn{2}{|c|}{0.697} & 0.010 & \\
\hline Female gender & -0.738 & 1.013 & 0.040 & 0.006 \\
\hline
\end{tabular}

As noted above, all five variables significantly affect survival and were therefore retained in the final model. In addition, the $P$-values associated with the proportional hazards assumptions indicated that donor blood type $\mathrm{AB}$, use of a ventilator, and female gender violate the proportional hazards assumption, indicating that the effects are not constant over time. Therefore Cox PH model may not be sufficient to capture the temporal changes of these covariate effects.

Figure 1 depicts changes in the covariate effects over time based on the final multivariable Gray PC-TVC model. The covariate effects in black solid lines are from Gray PCTVC model with 4 degrees of freedom for each variable, with pointwise $95 \%$ confidence intervals shaded in grey. For comparison, the estimated average covariate effects from Cox $\mathrm{PH}$ model are shown in red.

The most notable effect is that recipients who received livers from donors of blood type $\mathrm{AB}$ tend to have lower risks of death in the first two years, but then experience significantly higher risks afterwards. The Gray PC-TVC model shows that donor blood type $\mathrm{AB}$ has a strong nonproportional effect on posttransplant survival for recipients diagnosed with cancer, a finding that cannot be observed at all in the Cox $\mathrm{PH}$ model. (It is unclear why recipients who received livers from donors with $\mathrm{AB}$ blood type had better survival. The most obvious hypothesis is that this finding pointed to the benefit of exact matches between recipient and donor blood types. Unfortunately, the data do not support this hypothesis).

Ventilator use at time of transplant results in a strong decreasing trend. In the short term, patients who required ventilator support at time of transplant are sicker and have higher risks of death than those who do not, but the difference diminishes over time and these patients have better long-term survival. In contrast, results of the $\mathrm{Cox} \mathrm{PH}$ model suggest that ventilator use is not significant with average hazard ratio of $2.3\left(\mathrm{e}^{0.83}\right)$. Similarly, female recipients tend to have higher risk at the beginning, but better survival on the long run than male recipients. The effect of gender is marginally significant in the Gray PC-TVC model, but trivial in the Cox PH model. For other covariates like creatinine at time of transplant and positive CMV, Cox and Gray both report significance, but none of the effects varies over time. For these variables, as the proportional hazards assumption is not violated, it may be reasonable to use the Cox $\mathrm{PH}$ model. It is therefore of interest to consider an additional model allowing for both time-varying and time-invariant covariate effects. This was accomplished by a Gray PC-TVC model combining both linear terms and spline functions of covariates. Based on the previous results, we fit donor blood type, recipient gender, and ventilator usage as spline-based time-varying effect functions and positive CMV and serum creatinine as linear fixed effect covariates. The results are shown in Table 3. Compared with the results of our previous fitted Gray model (all covariate effects are time-varying), the estimated timevarying effects of covariates in the additional model are very similar. In addition, the estimated time-invariant effects of covariates also show similar magnitude as the coefficients obtained from the previous Cox model (all covariates are time-invariant).

Finally, we reran the multivariable Gray model with the same final set of covariates as in complete-case analysis based on the multiple imputed data. The results are shown in Table 4. Compared to the results in Table 2 (assumed MCAR), the magnitude and functional trend of estimated coefficients in Table 4 do not change noticeably. However, the effect of recipient gender becomes nonsignificant from $P=0.045$ to 0.084 . Figure 2 depicts the estimated covariate effects for gender when missing data was treated as MCAR Figure 2(a) and treated as MAR, Figure 2(b). Although $P$-value changes, the difference in patterns of the time-varying effects is not detectable.

\section{Discussion}

Liver transplant survival analyses often apply standard Cox $\mathrm{PH}$ model for estimation. In the present paper, we applied the Gray penalized spline method as an alternative to the Cox $\mathrm{PH}$ model to determine the important predictors and estimate their time-varying effects. The time-invariant effects were estimated as well using standard Cox PH model on the same set of predictors. We included only the pediatric patients with a diagnosis of cancer (primary liver malignancy) from the UNOS data. The results were reported graphically and numerically, showing key differences between two models. Donor $\mathrm{AB}$ blood type, ventilator use, and recipient gender 

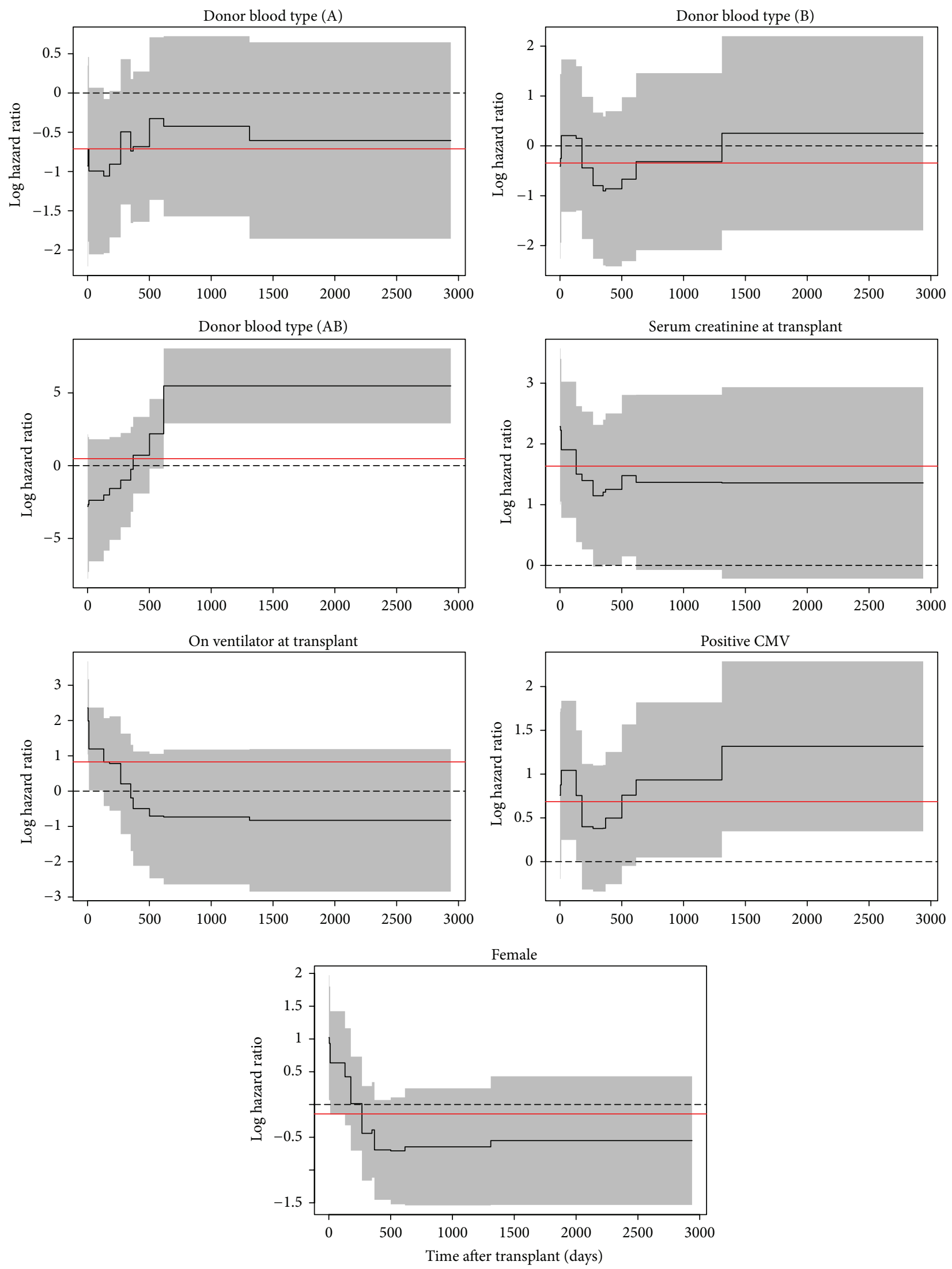

FIGURE 1: Time-varying covariate effects (black solid lines) with 95\% confidence intervals (shaded areas) are from the final Gray PC-TVC model with 4 degrees of freedom for each variable. The constant covariate effects (red solid lines) are estimated from the Cox PH model. 
TABLE 4: Estimated coefficients and test $P$ values using multiple imputed data.

\begin{tabular}{|c|c|c|c|c|}
\hline \multirow{2}{*}{ Covariate } & \multicolumn{2}{|c|}{ Cox PH } & \multicolumn{2}{|c|}{ Gray PC-TVC } \\
\hline & Log hazard ratio $(95 \% \mathrm{CI})$ & $P$ value & $P$ value & Nonprop $P$ value \\
\hline \multicolumn{5}{|l|}{ Donor blood type } \\
\hline A & $-0.608(-1.341,0.126)$ & 0.104 & 0.234 & 0.547 \\
\hline B & $-0.387(-1.577,0.803)$ & 0.524 & 0.694 & 0.533 \\
\hline $\mathrm{AB}$ & $0.486(-1.205,2.176)$ & 0.573 & 0.001 & 0.003 \\
\hline Serum creatinine $(\mathrm{mg} / \mathrm{dL})$ & $1.698(0.768,2.626)$ & $<0.001$ & $<0.001$ & 0.468 \\
\hline On ventilator & $0.842(-0.149,1.834)$ & 0.096 & 0.003 & 0.016 \\
\hline Positive CMV & $0.670(0.098,1.243)$ & 0.022 & 0.014 & 0.134 \\
\hline Female gender & $-0.178(-0.750,0.394)$ & 0.542 & 0.084 & 0.018 \\
\hline
\end{tabular}

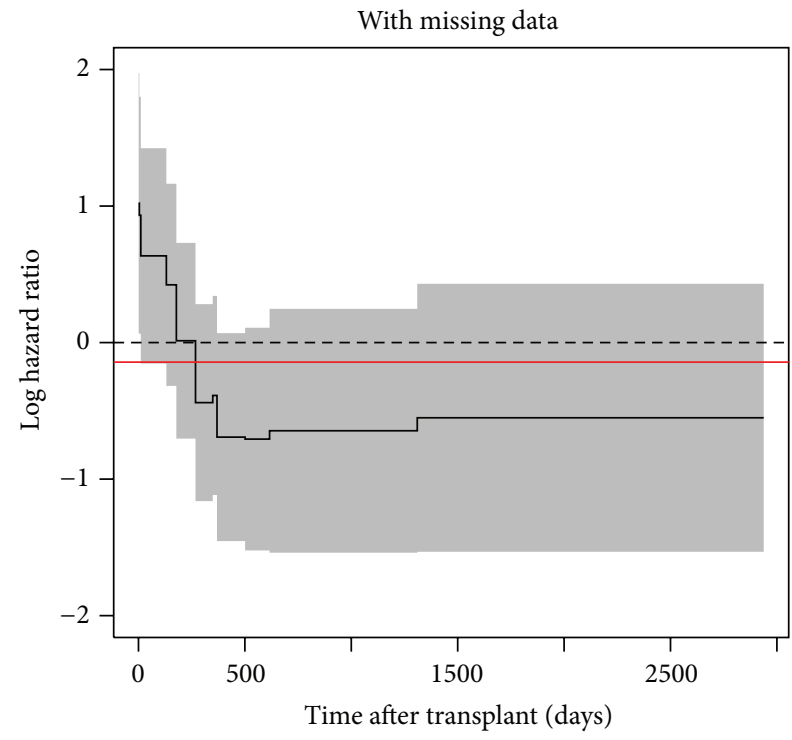

(a)

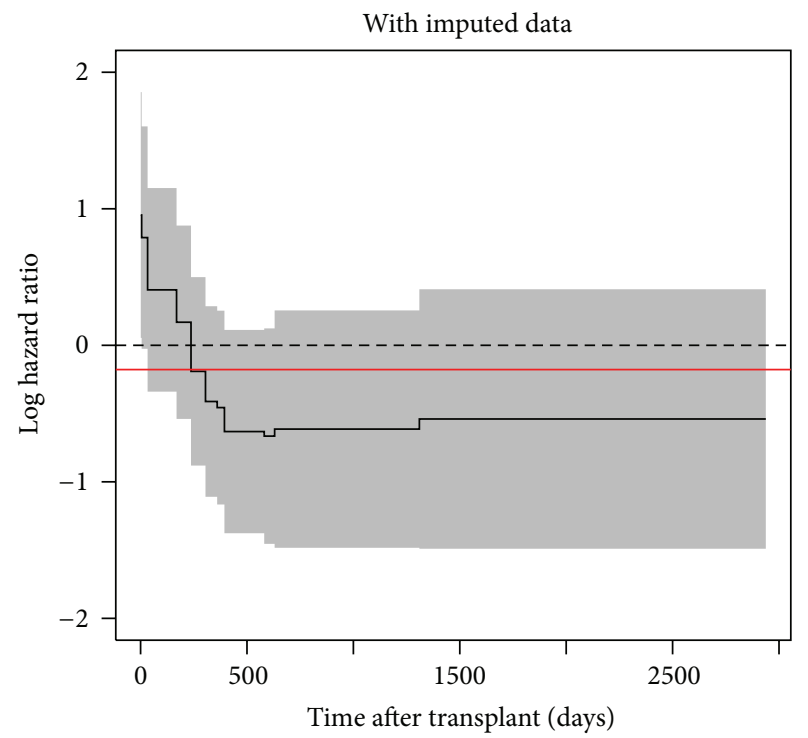

(b)

FIGURE 2: Time-varying covariate effects of recipient gender from the data with missing values (a) and with multiple imputation (b). The constant covariate effects (red solid lines) are estimated from each Cox PH model.

are significant in Gray PC-TVC model but not significant in Cox PH model. Furthermore, their effects vary over time after transplant. Although Cox $\mathrm{PH}$ model provided average estimates of coefficients, it failed to capture changes during observation period.

While the multiplicative hazards assumption holds without specifying the parametric form of baseline hazard function, Gray PC-TVC model has greater flexibility when the regression covariate effects change over time. It also can be used to check the proportional hazards assumption for the data. Many methods can be used to check the adequacy of a Cox model; however, no methods published can be used to check the overall goodness-of-fit for a Gray PCTVC model. Based on the method proposed by Kang [8], the graphical check of goodness-of-fit for the final Gray PCTVC model and Cox $\mathrm{PH}$ model using pseudoresiduals is presented in Figures 3 and 4, respectively. In the figures, the pseudoresiduals were calculated and plotted along with the lowess smoothed curves against the estimated survival rates at each of the nine preselected time points. Since the lowess smoothed curves of pseudoresiduals in Figure 3 stay around zero and are stable at most of time points (except time points 2 and 3) without any significant departure or tendency, we can conclude that the final multivariable Gray model shows a good fit in estimating posttransplant survival function. In Figure 4, however, the pseudoresiduals clearly illustrate departure from zero and some tendency. Therefore, final multivariable Cox model shows lack of fit to the data.

The main limitation of this analysis is the small sample size, which limits the generalizability of our findings and the set of covariates that could be considered. To examine the stability of the results given the uneven distribution of donor blood type and small sample sizes, we conducted sensitivity analysis. We first removed two observations with donor blood type $\mathrm{AB}$ and refit the final multivariable Gray PC-TVC and Cox $\mathrm{PH}$ models. The results showed that after removing these 
Pseudoresidual to assess goodness-of-fit test for Gray's time-varying coefficients model

$t 1$

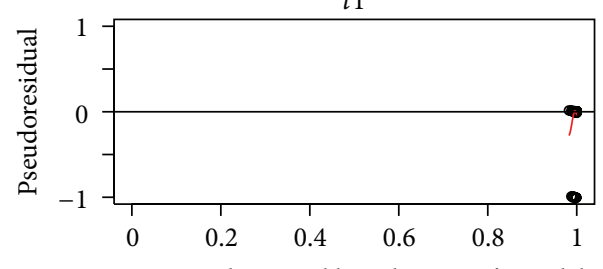

Estimated survival based on Gray's model

$t 4$

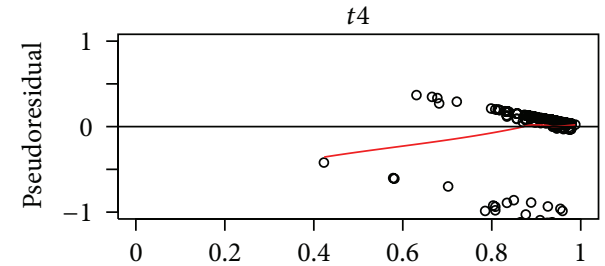

Estimated survival based on Gray's model

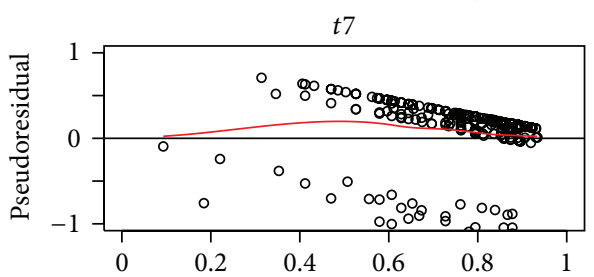

Estimated survival based on Gray's model

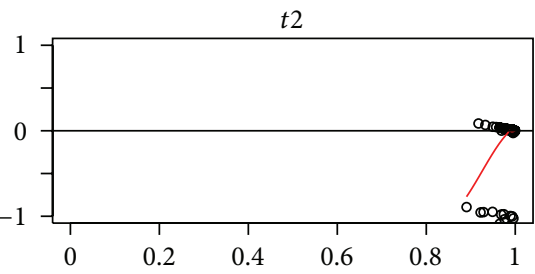

Estimated survival based on Gray's model

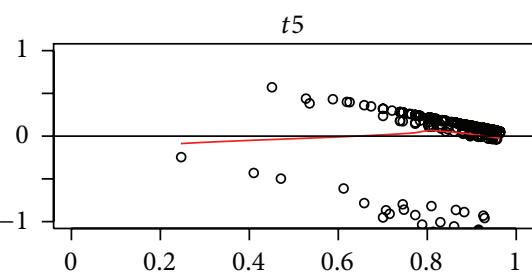

Estimated survival based on Gray's model

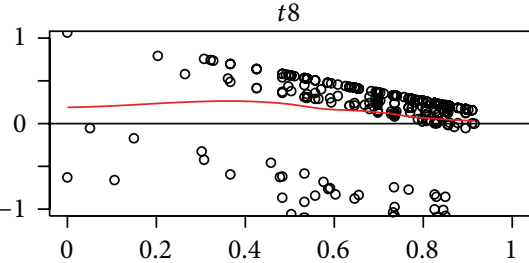

Estimated survival based on Gray's model

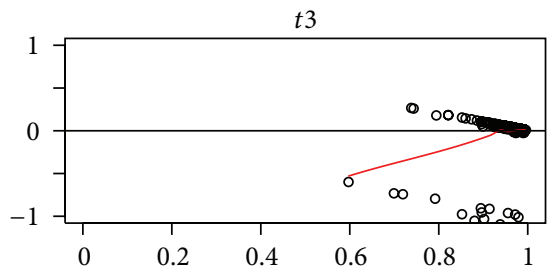

Estimated survival based on Gray's model

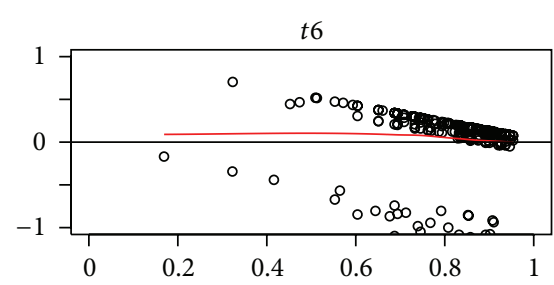

Estimated survival based on Gray's model

t9

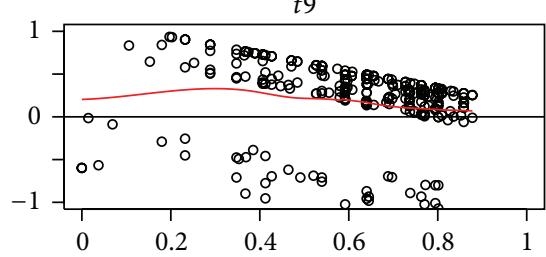

Estimated survival based on Gray's model

Figure 3: Goodness-of-fit test for Gray PC-TVC model using pseudoresiduals (dotted points) and lowess smoothed curves (red solid lines) against the estimated survival rates at each of the preselected time points.

Pseudoresidual to assess goodness-of-fit test for Cox PH model

$t 1$
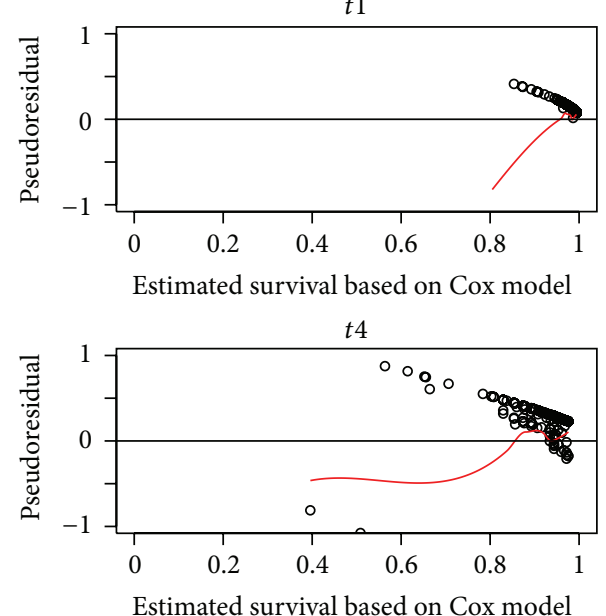

Estimated survival based on Cox model

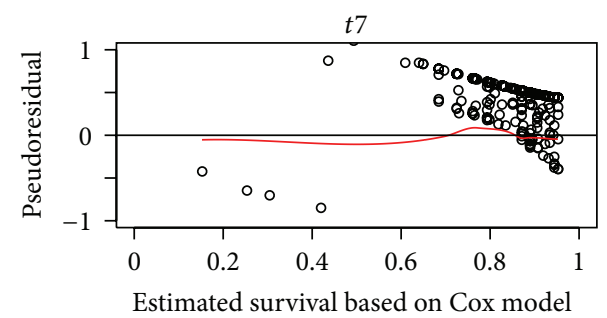

$t 2$
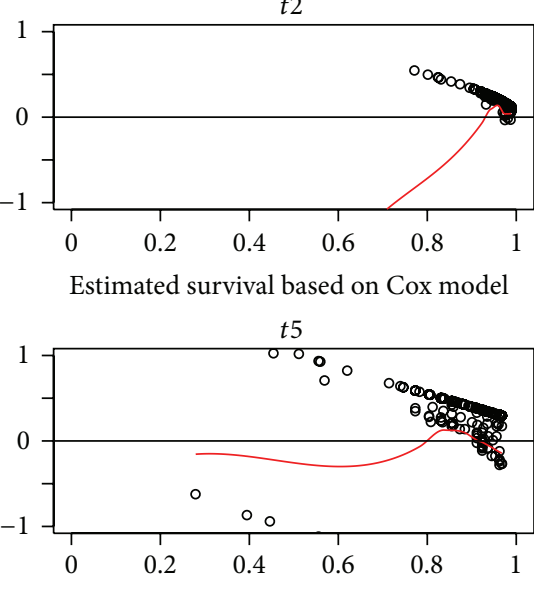

Estimated survival based on Cox model

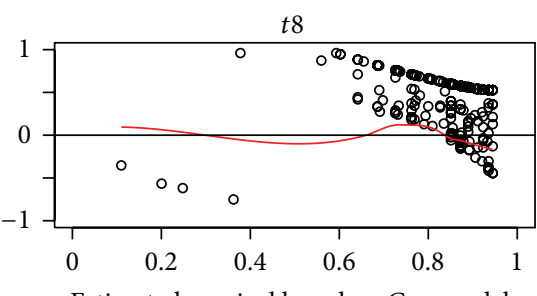

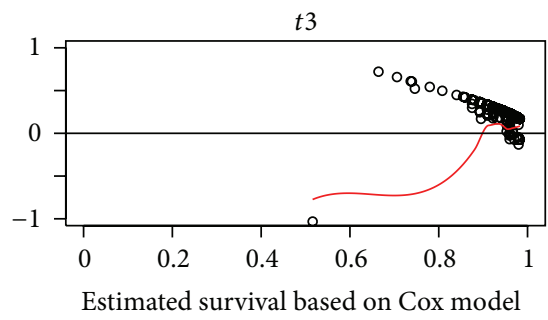

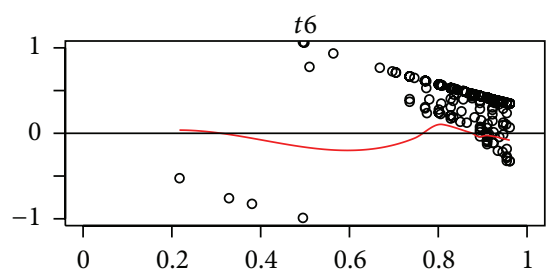

Estimated survival based on Cox model

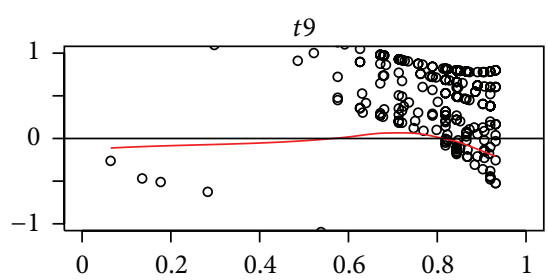

Estimated survival based on Cox model

Figure 4: Goodness-of-fit test for Cox PH model using pseudoresiduals (dotted points) and lowess smoothed curves (red solid lines) against the estimated survival rates at each of the preselected time points. 


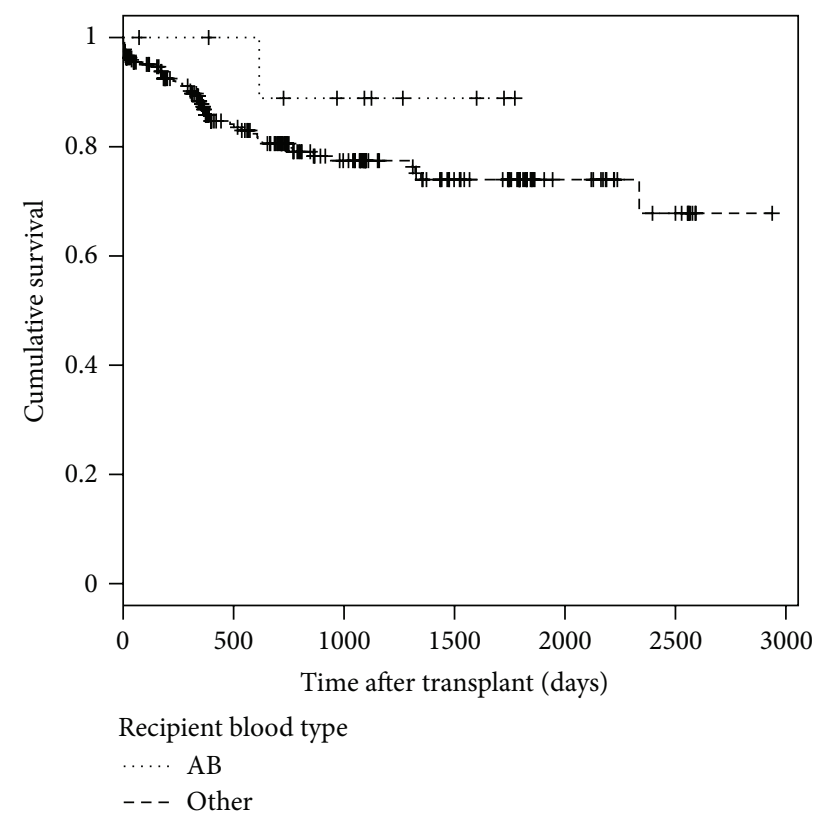

Figure 5: Kaplan-Meier survival estimates of the posttransplant survival time between recipients with blood type $A B$ and those with other blood types (logrank Chi-square statistic $=0.9264, P=0.336$ ).

two observations, recipient gender becomes nonsignificant from $P=0.045$ to $P=0.057$. After checking the time-varying pattern of the recipient gender effects (Appendix D), the effects are very similar and do not show noticeable difference.

However, transplant recipients with liver cancer were an appropriate cohort for meeting the primary goal of this paper, comparing Cox PH and Gray PC-TVC models and demonstrating the usefulness of more flexible approaches for estimating survival in some diseases. As the data here illustrated, using a Cox PH model in diseases where the proportional hazards assumptions are not satisfied can potentially lead to incorrect specifications and ignore the effect of important covariates.

\section{Conclusions}

While Cox $\mathrm{PH}$ model provided reasonable average results in estimating covariate effects on posttransplant survival, Gray model with piecewise constant penalized splines showed more details of how the effects change over time. An example of this is the effect of being on a ventilator at time of transplant. Requiring ventilator support indicates significant acute illness, often not directly related to liver disease. It therefore makes sense that the effect of being on a ventilator might dramatically affect early postoperative mortality but that the effect would decline over time as the reason for ventilator support was treated. Because the Cox PH model must average these effects to be constant over time, the higher early mortality "cancels" the lower later mortality and the effect of that variable is not significant in a Cox PH model.

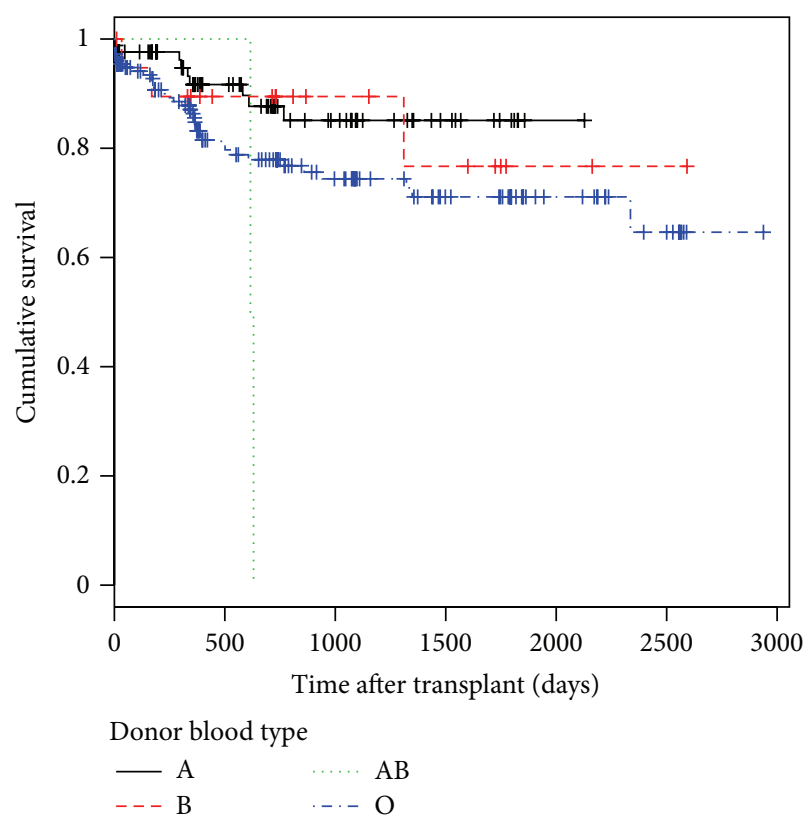

FIGURE 6: Kaplan-Meier survival estimates of the posttransplant survival by donor blood type (Tarone-Ware Chi-square statistic $=$ 8.0053, $P=0.046)$.

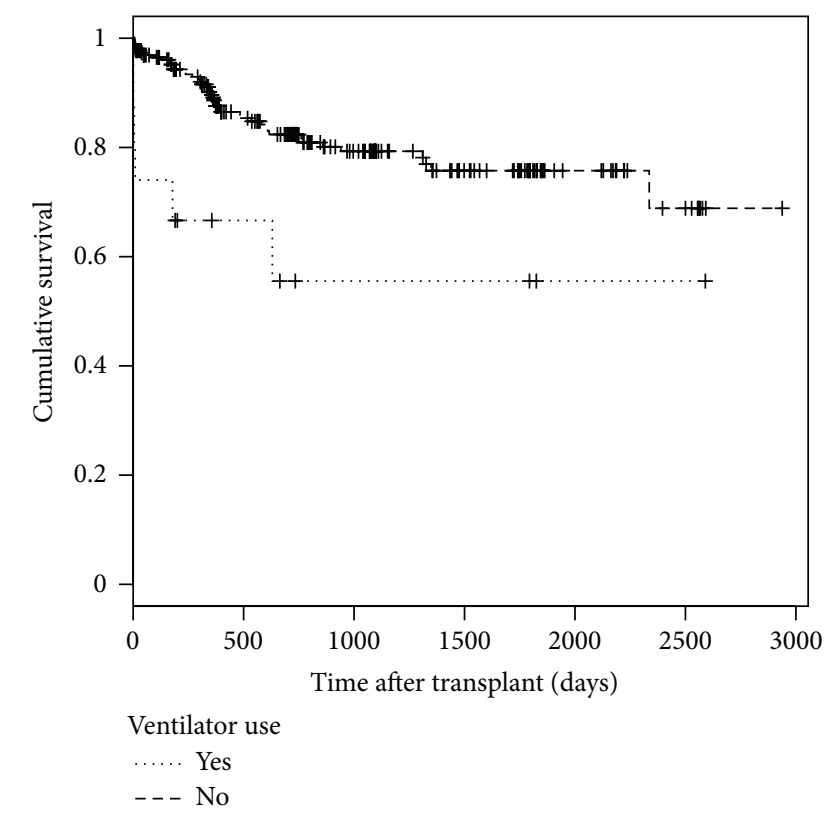

FIgURE 7: Kaplan-Meier survival estimates of the posttransplant survival time between recipients who used ventilator at time of transplant and those without using ventilator (Tarone-Ware Chisquare statistic $=11.4723, P=0.001)$.

Choosing the optimal time to perform transplantation is an essential way to improve patient survival. The timevarying coefficients model is more flexible than the traditional Cox PH model to estimate temporal changes that influence timing decisions and predictions about posttransplant survival. 
TABLE 5: Numbers of days of posttransplant survival for the 11 recipients with blood type AB.

\begin{tabular}{lcccccccccc}
\hline Survival time & 616 & 1124 & 387 & 968 & 726 & 1724 & 1600 & 1266 & 1092 & 1773 \\
Died & 1 & 0 & 0 & 0 & 0 & 0 & 0 & 0 & 0 & 0 \\
\hline
\end{tabular}

TABLE 6: Results of sensitivity analysis.

\begin{tabular}{|c|c|c|c|c|}
\hline \multirow{2}{*}{ Covariate } & \multicolumn{2}{|c|}{ Gray model $\mathrm{A}^{*}$} & \multicolumn{2}{|c|}{ Gray model B** } \\
\hline & Overall $P$ value & Nonprop $P$ value & Overall $P$ value & Nonprop $P$ value \\
\hline \multicolumn{5}{|l|}{ Donor blood type } \\
\hline A & 0.163 & 0.498 & 0.143 & 0.394 \\
\hline $\mathrm{B}$ & 0.679 & 0.480 & 0.695 & 0.500 \\
\hline $\mathrm{AB}$ & 0.001 & 0.001 & & \\
\hline Serum creatinine $(\mathrm{mg} / \mathrm{dL})$ & 0.001 & 0.527 & 0.001 & 0.545 \\
\hline On ventilator & 0.002 & 0.012 & 0.002 & 0.034 \\
\hline Positive CMV & 0.017 & 0.163 & 0.020 & 0.210 \\
\hline Female gender & 0.045 & 0.007 & 0.057 & 0.010 \\
\hline
\end{tabular}

* Previous final multivariable Gray model.

** Gray model with two observations removed.

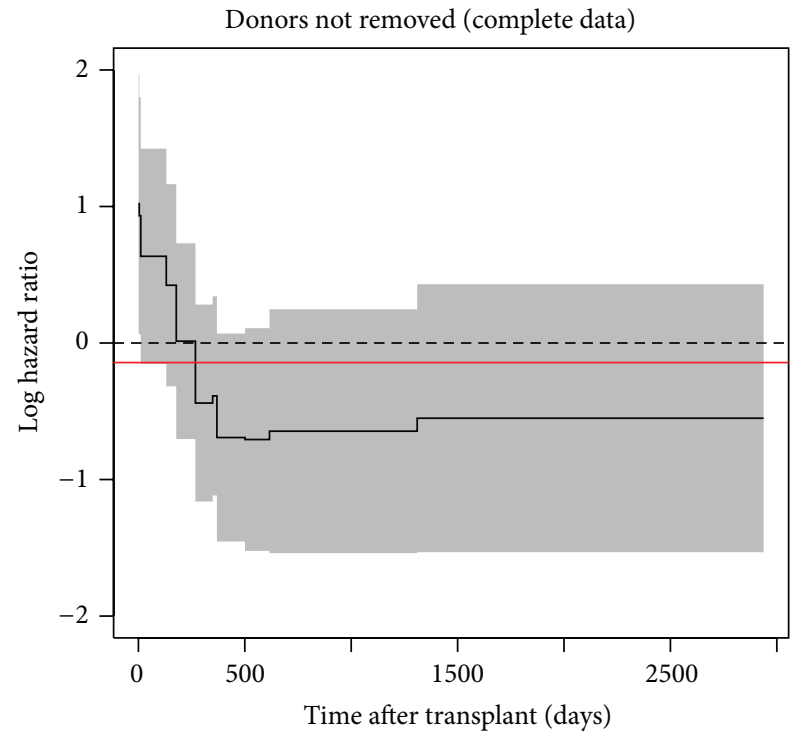

(a)

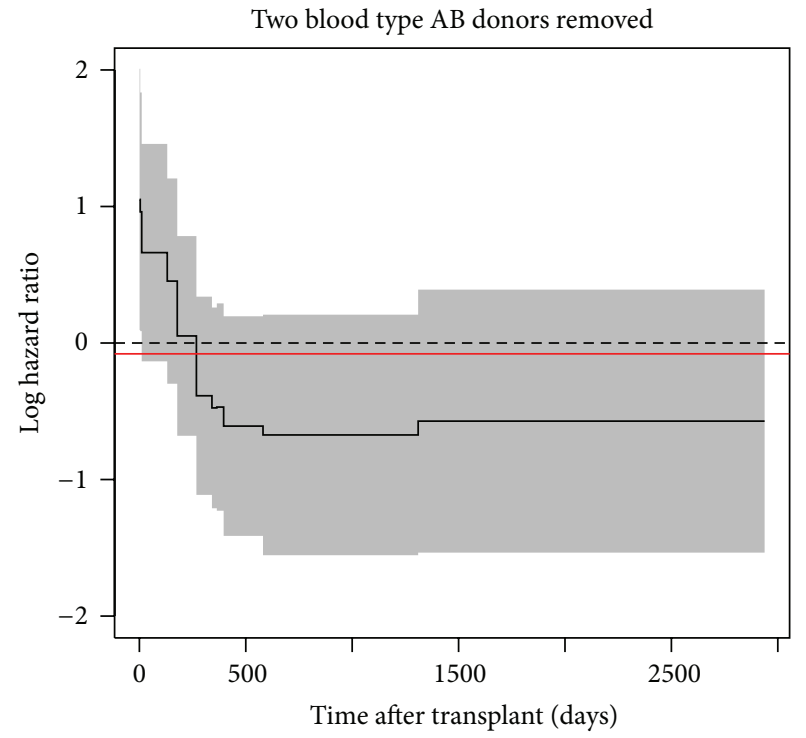

(b)

FiguRe 8

\section{Appendices}

\section{A. The Posttransplant Survival Functions for Recipients with Blood Type AB}

See Table 5 and Figure 5.

\section{B. The Posttransplant Survival Functions by Donor Blood Type}

See Figure 6.

\section{The Posttransplant Survival Functions for Ventilator Users and Nonusers at Time of Transplant}

See Figure 7.

\section{Sensitivity Analysis for the Impact of Extreme Distribution of Donor Blood Type on Covariate Effects}

See Table 6 and Figure 8. 


\section{References}

[1] J. C. Bucuvalas, L. Zeng, and R. Anand, "Predictors of length of stay for pediatric liver transplant recipients," Liver Transplantation, vol. 10, no. 8, pp. 1011-1017, 2004.

[2] P. J. Thuluvath, M. K. Guidinger, J. J. Fung, L. B. Johnson, S. C. Rayhill, and S. J. Pelletier, "Liver transplantation in the United States, 1999-2008: special feature," American Journal of Transplantation, vol. 10, no. 4, pp. 1003-1019, 2010.

[3] D. R. Cox, "Regression models and life-tables," Journal of the Royal Statistical Society B, pp. 187-220, 1972.

[4] R. J. Gray, "Flexible methods for analyzing survival data using splines, with applications to breast cancer prognosis," Journal of the American Statistical Association, vol. 87, no. 420, pp. 942951, 1992.

[5] B. Efron, "The efficiency of Cox's likelihood function for censored data," Journal of the American Statistical Association, vol. 72, no. 359, pp. 557-565, 1977.

[6] Z. Valenta and L. Weissfeld, "Estimation of the survival function for Gray's piecewise-constant time-varying coefficients model," Statistics in Medicine, vol. 21, no. 5, pp. 717-727, 2002.

[7] R. J. Gray, "Spline-based tests in survival analysis," Biometrics, vol. 50, no. 3, pp. 640-652, 1994.

[8] H.-J. Kang, Use of pseudo-observation in the goodness-of-fit test for Grey's time-varying coefficients model [M.S. thesis], Department of Biostatistics, Graduate School of Public Health, University of Pittsburgh, 2011. 


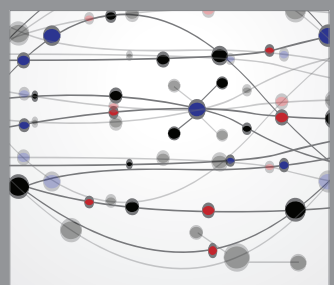

The Scientific World Journal
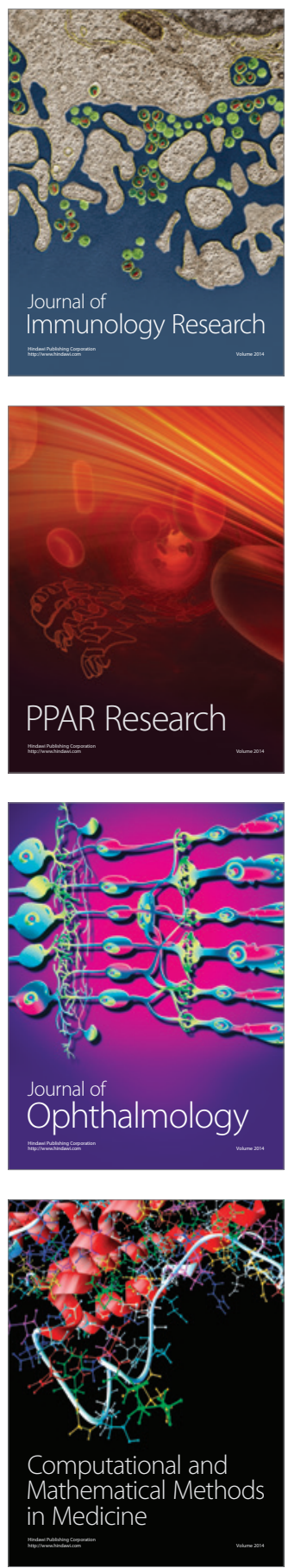

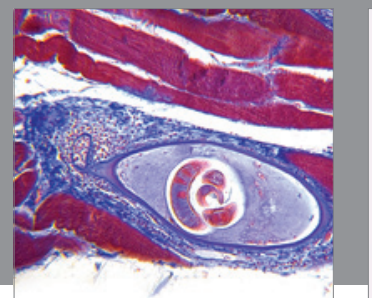

Gastroenterology

Research and Practice
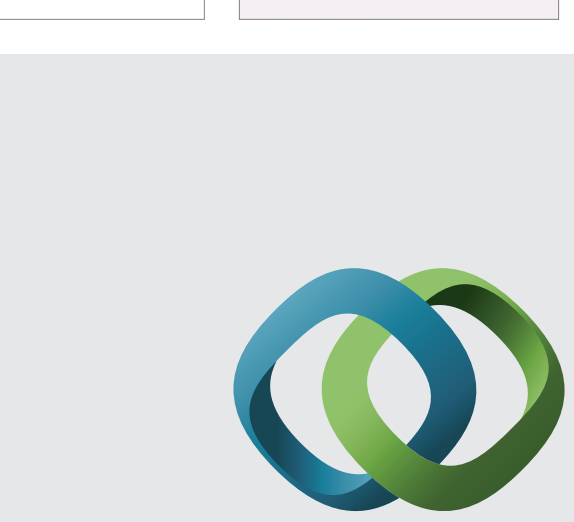

\section{Hindawi}

Submit your manuscripts at

http://www.hindawi.com
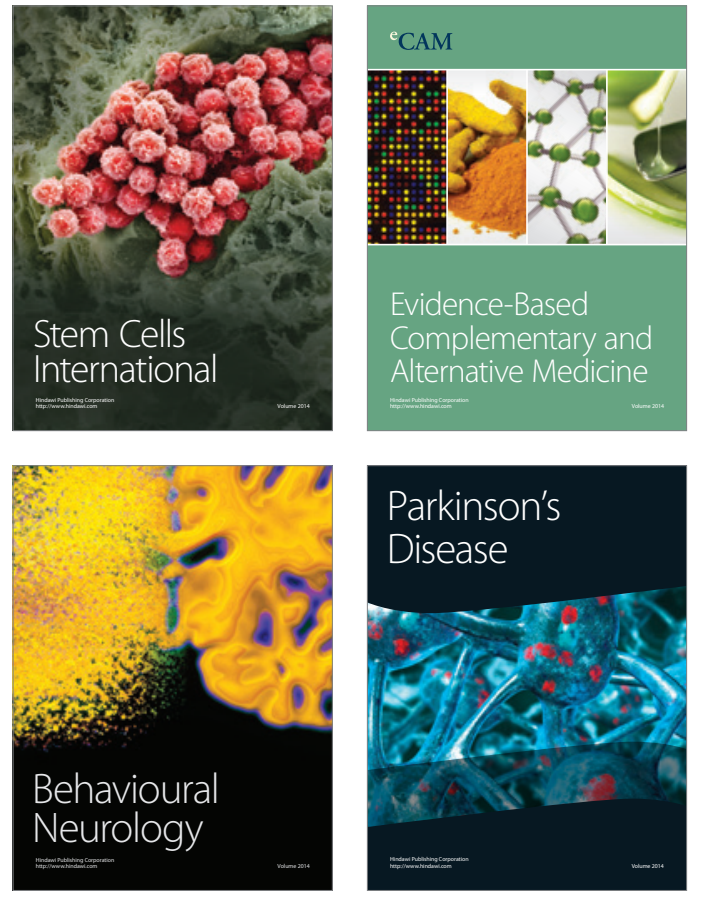
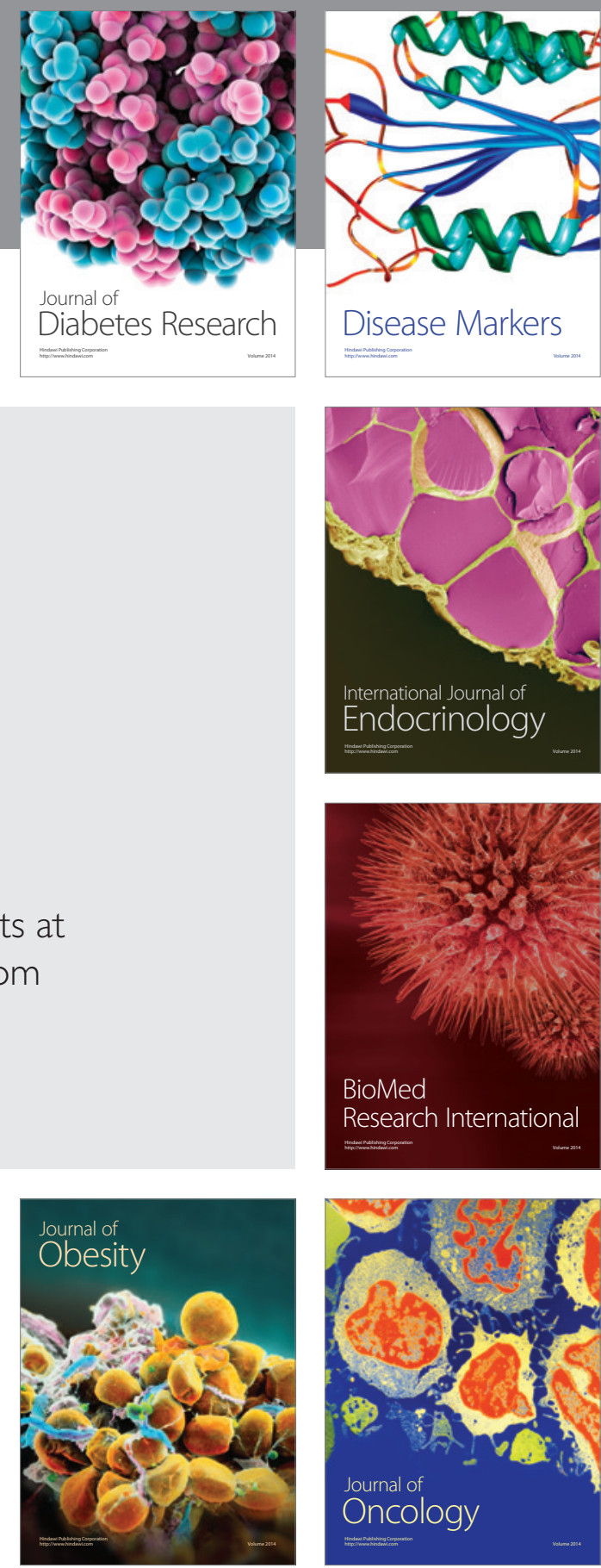

Disease Markers
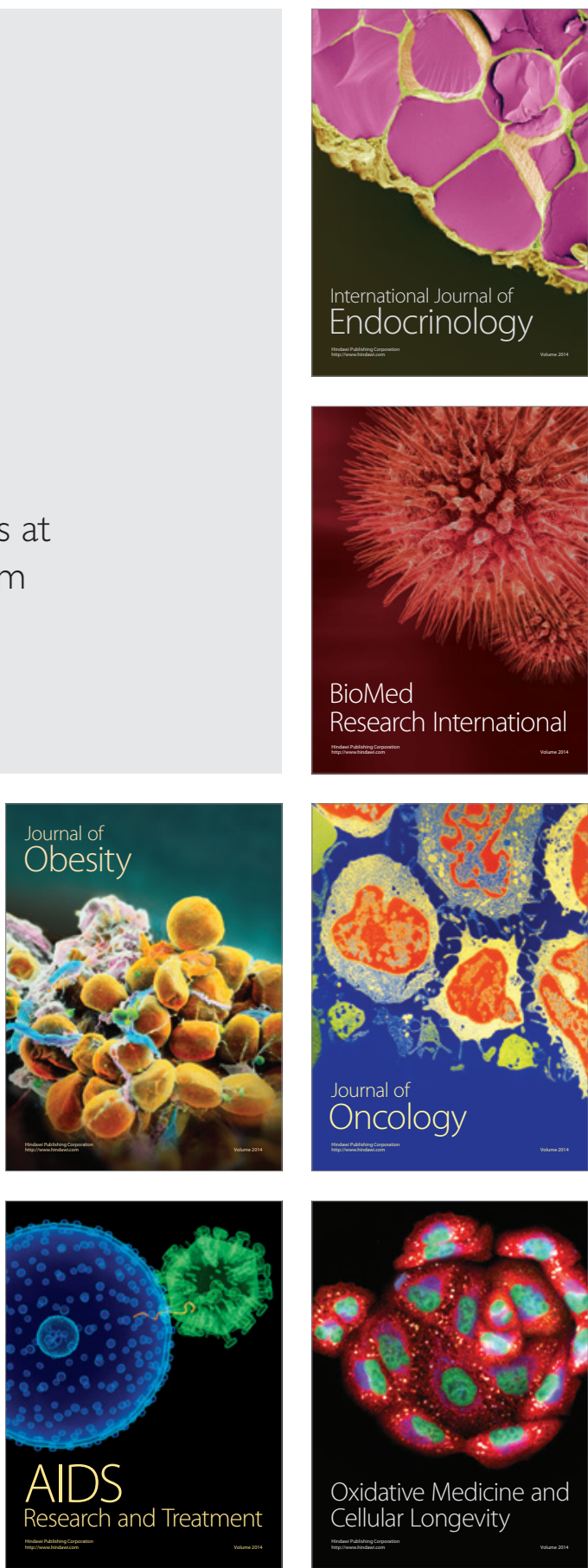